\title{
REVIEW \\ Psychosocial aspects of feeding children with neurodisability
}

\author{
GM Craig
}

The psychosocial support needs of parents considering a gastrostomy feeding tube for their disabled child are often overlooked, yet there is a growing body of evidence that attests to the decisional conflicts parents, often mothers, experience. This may be in addition to the stress associated with feeding a disabled child. The support needs of families and caregivers should be assessed, including the values parents attach to oral and tube feeding. Structured support should be embedded within the care pathway and both professionals, and service users, with appropriate training should be identified to ensure parental information needs, and any emotional, practical and financial issues are addressed.

European Journal of Clinical Nutrition (2013) 67, S17-S20; doi:10.1038/ejcn.2013.226

Keywords: gastrostomy; enteral nutrition; maternal health; coping behavior; social support; integrated health care

\section{INTRODUCTION}

A high proportion of children with neurodisability, such as those with severe cerebral palsy (CP), experience disorders of swallowing and feeding with implications for their nutrition, growth and overall health. ${ }^{1}$ Dysphagia, although often under-recognized in this population of children ${ }^{2}$ is common, with reported prevalences ranging from about one-fifth of children with $\mathrm{CP}$ of any degree, ${ }^{3}$ to $99 \%$ in children with severe CP and intellectual disability. ${ }^{4}$ Pulmonary aspiration is also common (where food or fluid enters the airway). ${ }^{5}$ Caregivers, usually mothers, report prolonged mealtimes, which can be experienced as stressful..$^{6}$ In some cases, caregivers spend in excess of three hours per day feeding with individual mealtimes lasting more than half an hour. ${ }^{6}$

Not surprisingly, mothers may feel that their everyday lives are dominated by feeding, particularly where a child refuses to feed from anyone else, which can be experienced as isolating, as mothers feel unable to leave their child, go out to work or socialize. The demands of care can be high in this population of children as they are frequently ill and require numerous visits to health professionals and periods of hospitalization. ${ }^{7}$ Frequent hospital appointments, because of the complexity of children's needs, and the lack of integrated services, in some cases, can also create additional demands on children and their caregivers, pointing to the importance of the role of key workers in coordinating children's care. ${ }^{8}$

Research into the benefits of gastrostomy tube feeding reports improvements in health and weight gain 7,9 and decreased stress, as experienced by caregivers. ${ }^{10,11}$ Parents also report a reduction in the amount of time spent feeding and ease of administering medication and liquids (by tube rather than orally). They generally worry less about their child's nutrition with the knowledge that they are getting sufficient via the tube. ${ }^{12}$ Despite the benefits, the suggestion of a gastrostomy feeding tube (GFT) can generate opposition for some families. ${ }^{13,14}$ For others, however, an alternative to feeding by mouth may come as a welcome relief. Occasionally, families may find their request for a gastrostomy tube is met with the advice to 'persevere' or 'try harder' from clinicians, as guidelines on the use of gastrostomy are underdeveloped. Research across six European countries, for example, has demonstrated considerable variation in the use of GFT and the age at which they are placed, which could be attributed, in part, to variations in clinical practice. ${ }^{15}$ Delaying surgery may add to the stress already experienced by caregivers. Ongoing assessment of parental psychosocial support needs and their ability to cope with oral/nasogastric tube feeding, with a view to moving toward feeding by a GFT, should form an essential aspect of clinical care. ${ }^{16,17}$

\section{SUPPORTING PARENTS THROUGH THE DECISION-MAKING PROCESS}

There is a growing body of literature, which highlights those factors influencing parental decision making in relation to the placement of a gastrostomy. This research emphasizes the importance of understanding the symbolic meanings of GFTs in relation to maternal identity, the maternal role, food, feeding, eating and child development. ${ }^{17-22}$

\section{FEEDING AND THE MOTHERING ROLE}

The suggestion of a GFT may challenge the 'good mother narrative' and signify maternal failure. Mothers may blame themselves for the child's inability to feed and poor growth or feel blamed by members of the extended family. Whereas a 'fat' baby is often a sign of good health in some cultures, ${ }^{23}$ a thin child may be associated with a neglectful and poor mother. This may have a particular resonance in cultures with a history of deprivation. ${ }^{24}$ Although parents may feel anxious about a child having an operation, the decision to elect for a GFT can be viewed as distinctive because feeding children as well as their growth and development are often deemed to be the responsibility of mothers. ${ }^{22}$

Caregivers may find feeding stressful but this does not always indicate their readiness to accept a GFT. Feeding may be described as 'difficult' or 'slow' or even akin to a 'war' or 'battle' reflecting the difficulties around feeding. However, some mothers also designate mealtimes as a 'special time' and a time for intimacy and closeness, ${ }^{17}$ which they fear they will lose if their children were fed by tube only. Tube feeding can signify many things and it is important to explore what parents feel they are 'giving up' versus what they are gaining, when they agree

School of Health Sciences, City University London, London, UK. Correspondence: Dr GM Craig, School of Health Sciences, City University London, Northampton Square, London EC1V OHB, UK.

E-mail: gill.craig.1@city.ac.uk

This supplement was derived from a meeting with experts facilitated by Josephine Garvey and Annemiek Goedhart, held in Orlando in 2012. 
to feeding by tube. Listening to parental narratives and the meanings they attach to feeding can provide important information on how best to offer support. For example, advice that 'special time' with children can be simulated through other activities including bathing, play and preparing for bedtime may assist mothers to reframe 'good mothering' and enjoy 'special time' with their children in ways other than through feeding.

\section{INVESTMENTS IN ORAL FEEDING: DISABILITY AND CHILD DEVELOPMENT}

There may be many reasons for parental investment in oral feeding, not least because of the assumed pleasure children derive from eating and the experience with different tastes. Underpinning parental desire for children to experience different types of food is the belief that eating orally is important for language acquisition or has developmental benefit. ${ }^{17}$ Children's ability to manage different textures may also be taken as evidence of their progress in achieving developmental milestones. A return to 'liquid food' with little opportunity to experiment with tastes and textures or for children to indicate their preference may be viewed as a regressive step in terms of children's development. ${ }^{25}$

Other compensatory experiences can be encouraged such as massage, kissing and touching while children are tube fed. Advice on mouthing toys, suitable for children's age and needs, which simulate mouthing experiences, can also be discussed with a speech and language therapist. ${ }^{26}$

The importance of the family meal and eating together is often mentioned by parents as a barrier to tube feeding, especially where mealtimes are seen as a time for conversation, inclusion and participation. Parents may need advice on how to manage tube feeding in the context of the family meal. For example, feeding the child at the table and encouraging play with feeding utensils. ${ }^{16}$

For some families, the suggestion of a gastrostomy may be received negatively, a new diagnosis that they have to come to terms with. ${ }^{17,27}$ It may signify permanence and hence further evidence of a child's disability. This may be challenging for parents coming to terms with a child's disability and trying to normalize family life. ${ }^{28}$ Some parents may deny that their child has a feeding difficulty altogether. In these cases, input from a psychologist, to discuss age appropriate feeding and offer emotional support, may assist parents to come to terms with the idea of feeding their child by tube in the future.

The suggestion of a GFT may be more acceptable to those families where children are already fed by a nasogastric tube, particularly in cases where a child has had a tube since birth. For these families, tube feeding may be seen as a natural progression or the next step. Although a nasogastric tube is only intended as a short-term solution, children may have been fed by nasogastric tube for many years, which is not ideal, and can place demands on both caregivers and children. Parents would benefit early on from a care plan with goals for children's feeding identified in the short, medium and long term with dates for review.

Parents are often anxious that their child will refuse to eat orally following surgery for a GFT, as some children begin to stop eating by mouth for reasons that are not entirely clear. In some cases, professionals may advise against oral feeding altogether because of the risk of pulmonary aspiration and the association with respiratory disease. ${ }^{29}$ Although some experts currently question the necessity of a complete nil-by-mouth regimen for the majority of children with $\mathrm{CP}$, other health care professionals may feel reluctant to take this risk. ${ }^{30}$ On the other hand, parents may be reluctant to accept professional advice that oral feeding is unsafe. $^{30}$ This can be controversial particularly where aspiration is 'silent', with no obvious, outward sign of distress. ${ }^{30}$ Advice to parents on the safety of oral feeding should be given within the context of the multidisciplinary team following appropriate investigations and assessment of risk. ${ }^{26}$
Although feeding by tube might make life easier for caregivers compared with oral feeding only, this may not be the best advice to give mothers who want to hear about the benefits to the child. It may help to stress some of the developmental benefits to children that parents report following gastrostomy insertion. For example, some parents notice that children are more alert and more able to concentrate and that there is more free time for parents to spend with siblings. Moreover, it is better to prepare families for some of the possible negative aspects of gastrostomy feeding as caregivers will have to learn new nursing procedures and there may be side-effects of surgery. ${ }^{7}$

Not all anxiety about tube feeding is psychological, however, and parents may have concerns about incurring additional costs associated with tube feeding and its affordability, particularly where equipment and feeds are not provided as part of a health plan or health service. Here, financial advice may be necessary.

\section{DEALING WITH THE UNEXPECTED: PREPARING CHILDREN AND FAMILIES}

Some families may only receive information about the practical side of managing tube feeding following surgery. However, research suggests that families would like the information sooner, including the impact of tube feeding on child and family in the context of their everyday lives. ${ }^{17}$

Information needs include: how to care for the tube; training carers to provide tube feeding in different contexts, such as school and residential care; how to explain tube feeding to siblings; practical tips for going on holiday abroad or short trips; and children's ability to engage in physical and therapeutic activities, such as swimming and physiotherapy. ${ }^{17}$ Providing checklists of items for families to research in advance of surgery may also prove useful. For example, research has suggested that children may be excluded from school or short-term residential care because of a lack of trained staff to feed children by tube. ${ }^{31}$ Families need to be prepared for these potential setbacks.

Parents might also express concerns that tube feeding is a form of 'force' feeding. They may be uncertain about whether to feed a child experiencing illness, particularly where loss of appetite is often the first sign. Parents can benefit from advice on how to manage these scenarios from other families or parental support organizations.

Appropriate post-operative care, both practical and emotional, is imperative. Families may experience problems obtaining the equipment or feeds and, in some cases, they can experience problems managing the equipment or caring for the stoma. ${ }^{31}$ Families should be provided with information signposting them to the relevant services and key people to contact before discharge from hospital.

Finally, parents may experience additional stress because of their interactions with the clinical services (number and frequency of contacts) and the complexity of care pathways. Parents may feel disempowered as professionals seemingly take 'charge' of children's care leaving parents as 'silent partners'. ${ }^{32}$ Clinicians may also use language that inadvertently reinforces blame and challenges mothers' parenting skills. Terms such as: 'failure to thrive' and 'malnourished' are best avoided. Actively involving parents as partners in decision making ${ }^{33}$ and valuing their expertise as feeders and carers can assist parents to feel less de-skilled in clinical encounters.

To summarize, families can be supported by managing their expectations, involving them to identify goals that are important to them, recognizing their expertise, building in time to discuss psychosocial aspects within the clinical care pathway and putting families in contact with other parents with experience of tube feeding. Ensuring adequate information on all aspects of gastrostomy feeding including the practical, social and emotional 
aspects is essential. ${ }^{31}$ (See Appendix for a list of supportive suggestions.)

This supplement is provided as a professional service by the Paediatric Division of Nutricia Advanced Medical Nutrition.

\section{CONFLICT OF INTEREST}

GM Craig has received payment from Nutricia Ltd for a lecture and travel expenses.

\section{REFERENCES}

1 Reilly S, Skuse D, Poblete X. Prevalence of feeding problems and oral motor dysfunction in children with cerebral palsy: a community survey. J Pediatr 1996; 129: 877-882.

2 Reilly S, Wisbeach A, Carr L. Assessing feeding in children with neurological problems. In: Southall A, Schwartz A (eds) Feeding Problems In Children a Practical Guide. Radcliffe Medical Press: Abingdon, Oxon, UK, 2000, pp 153-170.

3 Parkes J, Hill N, Platt MJ, Donnelly C. Oromotor dysfunction and communication impairments in children with cerebral palsy: a register study. Dev Med Child Neurol 2010; 52: 1113-1119.

4 Calis EA, Veugelers R, Sheppard JJ, Tibboel D, Evenhuis HM, Penning C. Dysphagia in children with severe generalized cerebral palsy and intellectual disability. Dev Med Child Neurol 2008; 50: 625-630.

5 Gisel EG, Patrick J. Identification of children with cerebral palsy unable to maintain a normal nutritional state. Lancet 1988; 1: 283-286.

6 Sullivan PB, Lambert B, Rose M, Ford-Adams M, Johnson A, Griffiths P. Prevalence and severity of feeding and nutritional problems in children with neurological impairment: Oxford Feeding Study. Dev Med Child Neurol 2000; 42: 674-680.

7 Craig GM, Carr LJ, Cass H, Hastings RP, Lawson M, Reilly S et al. Medical, surgical, and health outcomes of gastrostomy feeding. Dev Med Child Neurol 2006; 48 : 353-360.

8 Sloper P, Turner S. Service needs of families of children with severe physical disability. Child Care Health Dev 1992; 18: 259-282.

9 Sullivan PB, Juszczak E, Bachlet AM, Lambert B, Vernon-Roberts A, Grant HW. et al. Gastrostomy tube feeding in children with cerebral palsy: a prospective, longitudinal study. Dev Med Child Neurol 2005; 47: 77-85.

10 Sullivan PB, Juszczak E, Bachlet AM, Lambert B, Vernon-Roberts A, Grant HW et al. Gastrostomy tube feeding in children with cerebral palsy: a prospective, longitudinal study. Dev Med Child Neurol 2005; 47: 77-85.

11 Avitsland TL, Faugli A, Pripp AH, Malt UF, Bjørnland K, Emblem R. Maternal psychological distress and parenting stress after gastrostomy placement in children. J Pediatr Gastroenterol Nutr 2012; 55: 562-566.

12 Sullivan PB, Juszczak E, Bachlet AM, Thomas AG, Lambert B, Vernon-Roberts A et al. Impact of gastrostomy tube feeding on the quality of life of carers of children with cerebral palsy. Dev Med Child Neurol 2004; 46: 796-800.

13 Sleigh G, Brocklehurst P. Gastrostomy feeding in cerebral palsy: a systematic review. Arch Dis Child 2004; 89: 534-539.

14 McGrath SJ, Splaingard ML, Alba HM, Kaufman BH, Glicklick M. Survival and functional outcome of children with severe cerebral palsy following gastrostomy. Arch Phys Med Rehabil 1992; 73: 133-137.

15 Dahlseng MO, Andersen GL, DA, Graca Andrada M, Arnaud C, Balu R, De la Cruz J et al. Gastrostomy tube feeding of children with cerebral palsy: variation across six European countries. Dev Med Child Neurol 2012; 54: 938-944.
16 Bond $\mathrm{P}$, Moss $\mathrm{D}$. Best practice in nasogastric and gastrostomy feeding in children. Nurs Times 2003; 99: 28-30.

17 Craig GM, Scambler G, Spitz L. Why parents of children with neurodevelopmental disabilities requiring gastrostomy feeding need more support. Dev Med Child Neurol 2003; 45: 183-188.

18 Mahant S, Jovcevska V, Cohen E. Decision-making around gastrostomy-feeding in children with neurologic disabilities. Pediatrics 2011; 127: e1471-e1481.

19 Sleigh G. Mothers' voice:a qualitative study on feeding children with cerebral palsy. Child Care Health Dev 2005; 31: 373-383.

20 Thorne SE, Radford MJ, McCormick J. The multiple meanings of long-term gastrostomy in children with severe disability. J Pediatr Nurs 1997; 12: 89-99.

21 Petersen MC, Kedia S, Davis P, Newman L, Temple C. Eating and feeding are not the same: caregivers' perceptions of gastrostomy feeding for children with cerebral palsy. Dev Med Child Neurol 2006; 48: 713-717.

22 Craig GM, Scambler G. Negotiating mothering against the odds: gastrostomy tube feeding, stigma, governmentality and disabled children. Soc Sci Med 2006; 62: 1115-1125.

23 Winstock A. The Practical Management of Eating and Drinking Difficulties in Children. Speechmark Publishing Ltd: Bicester, Oxon, UK, 2001.

24 Helman C. Culture, Health and IIIness. 5th edn, Hodder Arnold: London, UK, 2007.

25 Craig GM. Mother Knows Best: Gastrostomy Feeding in Disabled Children -Professional and Parental Discourses. PhD Thesis. University of London: UK, 2004.

26 Starr S. Management of paediatric feeding problems. In: Cichero JAY, Murdoch BE (eds) Dysphagia Foundation, Theory and Practice. Wiley: Chichester, UK, 2006, pp 487-539.

27 Petersen MC, Kedia S, Davis P, Newman L, Temple C. Eating and feeding are not the same: caregivers' perceptions of gastrostomy feeding for children with cerebral palsy. Dev Med Child Neurol 2006; 48: 713-717.

28 Thorne SE, Radford MJ, McCormick J. The multiple meanings of long-term gastrostomy in children with severe disability. J Pediatr Nurs 1997; 12: 89-99.

29 Cass H, Wallis C, Ryan M, Reilly S, McHugh K. Assessing pulmonary consequences of dysphagia in children with neurological disabilities: when to intervene? Dev Med Child Neurol 2005; 47: 347-352.

30 Craig G, Higgs P. Risk owners and risk managers: dealing with the complexity of feeding children with neurodevelopmental disability. Health Risk Soc 2012; 14: 627-637.

31 Townsley R, Robinson C. More than just a health issue: a review of current issues in the care of enterally fed children living in the community. Health Soc Care Commun 1999; 7: 216-224.

32 Brett J. The experience of disability from the perspective of parents of children with profound impairment:is it time for an alternative model of disability. Disabil Soc 2002; 17: 825-843.

33 Dale N. Working with Families of Children with Special Needs: Partnership and Practice. Routledge: London, UK, 1996.

34 Southhall A. Family and wider system perspectives. In: Southall A, Schwartz A (eds) Feeding Problems in Children: a Practical Guide. Radcliffe Medical Press: Abingdon, Oxon, UK, 2000, pp 103-120.

35 Baird G, McConachie H, Scrutton D. Parents' perceptions of disclosure of the diagnosis of cerebral palsy. Arch Dis Child 2000; 83: 475-480.

(1) $($ This work is licensed under a Creative Commons AttributionNonCommercial-NoDerivs 3.0 Unported License. To view a copy of this license, visit http://creativecommons.org/licenses/by-nc-nd/3.0/ 


\section{APPENDIX}

\author{
SUGGESTIONS FOR SUPPORTING FAMILIES* \\ Who should be involved? ${ }^{26}$ \\ Parents \\ Child \\ Consultant pediatrician \\ Gastroenterologist/surgeon \\ Speech and language therapist \\ Dietician \\ Occupational therapist \\ Clinical nurse specialist \\ Psychologist \\ Key worker (coordinate complex care) \\ Advocate
}

\section{PREPARING THE ENVIRONMENT FOR A DISCUSSION OF FEEDING}

Discuss somewhere private without interruptions

Provide privacy for parents afterward

Ensure interpreters available where necessary (book in advance)

\section{COMMUNICATING NEED FOR TUBE FEEDING}

Introduce members of the multi-disciplinary team (MDT) and their roles

Provide clear information

Manner-honest, warm and sympathetic

Positive tone (avoid 'I am afraid', 'unfortunately')

Avoid language that communicates blame

Explore parental narratives and meanings they attach to tube and oral feeding including any investments in oral feeding

Avoid a sole focus on food and weight and ask about feeding and relationships ${ }^{34}$

\section{INVOLVING PARENTS AND CHILDREN IN DECISION MAKING}

Involve parents and child in all aspects of decision making Involve children who may be able to communicate through a communication aid or discuss with parents how best to communicate with the child depending on age and severity of disability

Discuss a range of options for feeding with appropriate timelines for review

Explain the role and purpose of tests and investigations in informing decision making

Invite parents to ask questions throughout the appointment (not at the end)

Give parents time to think about questions (provide pauses)

Encourage parents to express their concerns

Acknowledge and value the expertise of parents in caring and feeding children

Explore other sources of stress the family may be experiencing, for example, family tensions, financial difficulties and other care-giving demands (responsibility for caring for other members of the family, or sick and disabled children)

Explore parental support mechanisms. Ask what assistance they feel they could benefit from. Build on existing strengths.

\section{CONSOLIDATING (ADAPTED FROM BAIRD ET AL. ${ }^{35}$ ON DISCLOSURE OF A DIAGNOSIS OF CP)}

Give a summary of discussion and outcome in writing, including parental views

Use child-friendly aids to assist communication with children, for example, storybooks with pictures of children with a gastrostomy or a gastrostomy demonstration doll

Offer an introduction to another child, parent or support group

Offer another (flexible) appointment to discuss GTF

Offer to talk to other family members

Signpost to other information and resources on GTF and the surgical procedure, parents may want to see examples of different types of gastrostomy

Offer appointment with the clinical nurse specialist and/or psychologist

\section{COORDINATING CARE}

Identify named key worker

Integrated care pathways

Guidelines that link community and specialist services ${ }^{17}$

\section{POST-SURGERY DISCHARGE PLANNING ${ }^{16}$}

Ensure sufficient supervised practice in administering feeds Ensure caregiver is confident in using and cleaning the equipment before discharge

Provide instruction on care for the stoma and whom to contact for advice

Give information on how to store feeds

Provide a named contact for coordinating supplies of equipment and feeds

Provide a named contact in the event of the tube becoming blocked or if parents experience difficulties with the equipment

\section{FOLLOW-UP}

Ensure appropriate clinical and dietetic input Offer support in reinstating oral feeding where appropriate Give advice on oral/dental hygiene

Offer psychosocial support

*Given that there are no guidelines on how best to support families considering a gastrostomy for their disabled child, a number of sources drawing on the wider literature have been used and adapted to inform these suggestions, which may be applicable to a wider range of children with feeding disabilities beyond the $\mathrm{CP}$ population. 